\title{
A Log-Weighted Power Function Distribution and Its Statistical Properties
}

\author{
Rasha Mohamed Mandouh ${ }^{1}$, Mahmoud Abdel-Ghaffar Mohamed ${ }^{1}$ \\ ${ }^{l}$ Department of Mathematical Statistics, Faculty of Graduate Studies for Statistical \\ Research, Cairo University, Egypt
}

\begin{abstract}
The Power function distribution is a flexible life time distribution that has applications in finance and economics. It is, also, used to model reliability growth of complex systems or the reliability of repairable systems. A new weighted Power function distribution is proposed using a logarithmic weight function. Statistical properties of the weighted power function distribution are obtained and studied. Location measures such as mode, median and mean, reliability measures such as reliability function, hazard and reversed hazard functions and the mean residual life are derived. Shape indices such as skewness and kurtosis coefficients and order statistics are obtained. Parametric estimation is performed to obtain estimators for the parameters of the distribution using three different estimation methods; namely: the maximum likelihood method, the L-moments method and the method of moments. Numerical simulation is carried out to validate the robustness of the proposed distribution.
\end{abstract}

Keywords: Log-weighted power function distribution, weighted distributions, survival function, hazard rate function, order statistics, maximum likelihood, moments, L-moments.

\section{Introduction}

The theory of weighted distributions provides a collective access to the problems of model specification and data interpretation. It provides a technique for fitting models to the unknown weight functions when samples can be taken both from the original distribution and a developed distribution. Fisher [8] introduced the basic idea of the concept of weighted distributions when he studied the effect of methods of ascertainment upon estimation of frequencies. In extending the basic ideas of Fisher, Rao [21] introduced a unified theory of weighted distributions and formulated it in general terms in connection with modeling statistical data where the standard distributions were not found to be appropriate. He identified various situations that can be modeled by weighted distributions. 
To introduce the concept of a weighted distribution, suppose $\mathrm{X}$ is a non-negative random variable with probability density function (pdf) $f(x ; \theta)$, where the parameter is $\theta \in \Omega(\Omega$ is the parameter space). Suppose a realization $\mathrm{x}$ of $\mathrm{X}$ under $\mathrm{f}(\mathrm{x} ; \theta)$ enters the investigator's record with probability proportional to $w(x, \beta)$ which is a nonnegative weight function with parameter $\beta$. So, the recorded $x$ is not an observation on $\mathrm{X}$, but on the random variable $\mathrm{Z}$, having such pdf

$$
\mathrm{g}(\mathrm{z} ; \theta, \beta)=\frac{\mathrm{w}(\mathrm{z}, \beta) \mathrm{f}(\mathrm{z}, \theta)}{\mathrm{W}}
$$

where $\mathrm{W}=\mathrm{E}[\mathrm{w}(\mathrm{x}, \beta)]$ is the normalizing factor obtained to make the total probability equal to unity. The random variable $\mathrm{Z}$ is called the weighted version of $X$, and its distribution in relation to that of $X$ is called the weighted distribution with weight function $w(x, \beta)$, Patil [20]. Different models can be obtained depending upon the choice of the weight function $\mathrm{w}(\mathrm{x})$.

The concept of weighted distributions has been employed in a wide variety of applications in reliability and survival analysis, analysis of family data, metaanalysis, ecology, medicine, and forestry. The contributions of researchers to the weighted distributions vary between single and double weight distributions. Shaban and Boudrissa [24] presented a length-biased version of the Weibull distribution. Gupta and Kundu [9] developed the weighted exponential (WE) distribution as a lifetime model. Levia, et al. [15] developed a length-biased version of the Birnbaum-Saunders [BS] distribution with applications in water quality. Kersey [14] presented the size-biased inverse Weibull distribution. Shi, et al. [25] presented the theoretical properties of weighted generalized Rayleigh distribution. Hussian [13] presented the weighted inverted exponential distribution as a generalized version of the inverted exponential distribution. Seenoi, et al. [23] developed a length-biased version of the exponentiated inverted Weibull (EIW) distribution. Dey, et al. [7] and Nasiru [17] presented different forms of the Weighted Weibull Distribution. Mahmoud, et al. [16] developed the weighted Quasi-Lindley distribution and weighted Lomax distribution. Bashir and Rasul [3] introduced a new weighted Rayleigh distribution named area-biased Rayleigh distribution. Al-kadim and Hantoosh [2] presented the double weighted exponential distribution (DWED). Ahmed and Ahmed [1] presented double weighted Rayleigh distribution (DWRD) version. Saghir and Saleem [22] presented a new version of the double weight Inverse Weibull (DWIW) distribution.

The Power function distribution has been used over the past decades for modeling data in several scientific fields such as engineering, reliability and biological studies. It is a flexible life time distribution that may offer a suitable fit to some sets of failure data. Suppose $\mathrm{X}$ is a continuous random variable following the Power function distribution with a pdf and a cdf as follows:

$$
f(x ; \alpha, \beta)=\frac{\alpha x^{\alpha-1}}{\beta^{\alpha}}, \quad 0<x<\beta, \quad \alpha>0
$$




$$
F(x ; \alpha, \beta)=\left(\frac{x}{\beta}\right)^{\alpha}, \quad 0<x<\beta, \quad \alpha>0
$$

where $\alpha$ and $\beta$ are shape and scale parameters respectively.

Some generalizations of the Power function distribution have been proposed. Among these distributions; the beta power function distribution presented by [6], Weibull power function distribution introduced by [26], Kumaraswamy power function distribution presented by [18], the modified power function distribution studied by [19], the exponentiated Kumaraswamy power function distribution investigated by [4], the transmuted Weibull power function distribution introduced by [10], and the exponentiated Weibull power function distribution presented by [11].

\section{Log-weighted Power Function Distribution}

Using the Power function distribution presented above as a base distribution, a new weighted distribution is introduced with a weight function, $\mathrm{w}(\mathrm{x})=-\log (\mathrm{x})$. The normalizing factor $\mathrm{W}$ is given by $\mathrm{W}=\mathrm{E}(-\log (\mathrm{x}))$ and it is obtained as follows:

$$
W=E(-\log (x))=\int_{0}^{\beta}(-\log (x)) \frac{\alpha x^{\alpha-1}}{\beta^{\alpha}} d x
$$

Integrating by parts, we obtain:

$$
\mathrm{W}=(1-\alpha \log (\beta))
$$

Applying equation (1) using the Power function distribution as a base distribution, a new weighted distribution called the Log-weighted Power function distribution is obtained. Its pdf and cdf are given by:

$$
\begin{array}{lll}
\mathrm{g}(\mathrm{y} ; \alpha, \beta)=\frac{\alpha^{2} \mathrm{y}^{\alpha-1}(\log \mathrm{y})}{\beta^{\alpha}(\alpha \log \beta-1)}, & 0<\mathrm{y}<\beta<1, & \alpha>0 \\
\mathrm{G}(\mathrm{y} ; \alpha, \beta)=\frac{\mathrm{y}^{\alpha}(1-\alpha \log \mathrm{y})}{\beta^{\alpha}(1-\alpha \log \beta)}, & 0<\mathrm{y}<\beta<1, & \alpha>0
\end{array}
$$

In addition, as the Power function distribution could be obtained from the Pareto distribution using the transformation $Z=\frac{1}{X}$, the Log-weighted Power function distribution can be obtained from the Log-weighted Pareto distribution using the same transformation. 


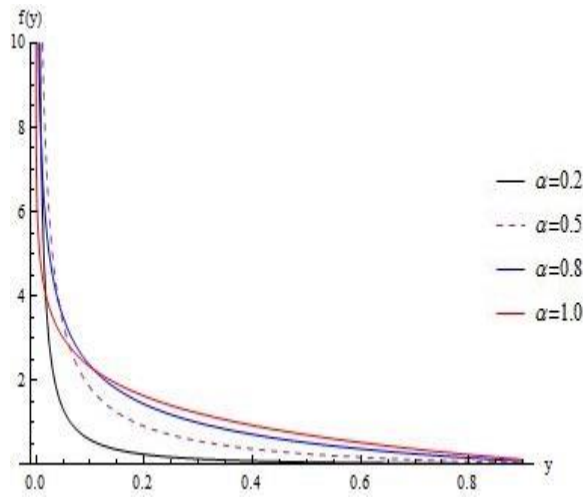

(a) $\alpha \leq 1, \beta=0.9$

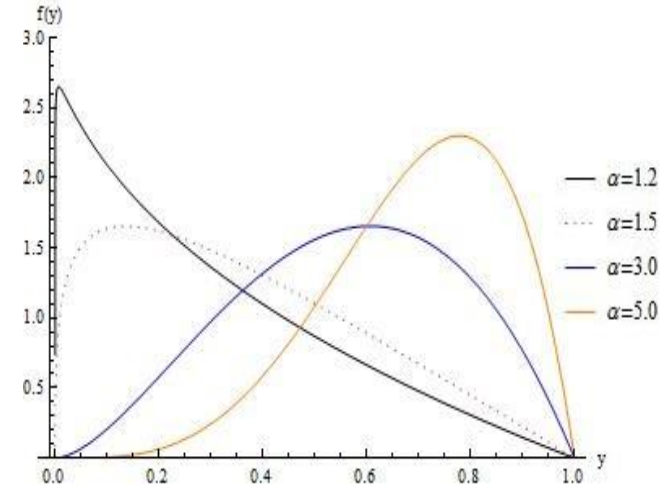

(b) $\alpha>1, \beta=1.0$

Figure 1. The LWPF pdf at selected values of $\alpha$

From figure 1, it is observed that the pdf is strictly decreasing when the value of $\alpha \leq 1$, but when $\alpha>1$, the pdf is a unimodal function. In addition, as the value of $\alpha$ increases, the function turns from a right skewed function to a left skewed function.

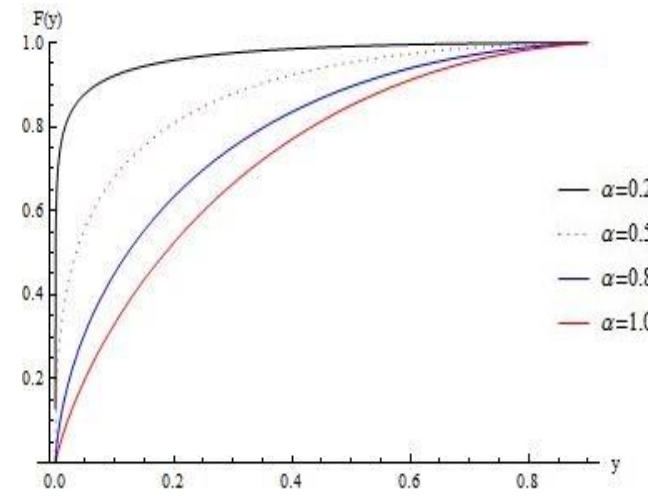

(a) $\alpha \leq 1$

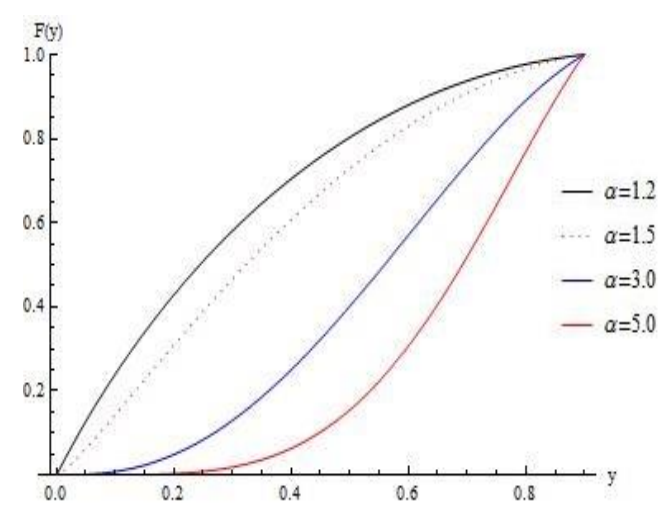

(b) $\alpha>1$

Figure 2. The LWPF cdf at $\beta=0.9$ and selected values of $\alpha$

From figure (2), one can observe that the cdf increases rapidly for small values of $\alpha$, but it increases slowly for large values of $\alpha$. In other words, the rate of increasing decreases as the value of $\alpha$ increases.

\subsection{The Mode and Quantiles}

The Log-weighted Power function density is a unimodal function and its modal value is achieved at $y=e^{-\left(\frac{1}{\alpha-1}\right)}$. On the other hand, there is no closed form for the quantiles, but it can be obtained numerically by using the following relation:

$$
p=F\left(q_{p}\right)=\frac{q^{\alpha}(1-\alpha \log q)}{\beta^{\alpha}(1-\alpha \log \beta)}, \quad 0<y<\beta<1, \alpha>0
$$

\subsection{Reliability measures}

The reliability function $\mathrm{R}(\cdot)$, the hazard rate function $\mathrm{h}(\cdot)$, the reversed hazard 
rate $\lambda(\cdot)$ and the mean residual life $\mathrm{m}(\cdot)$ of the LWPF distribution are given as follows:

\section{The reliability function:}

Reliability has become a major concern in almost all fields. The more reliable a component is, the more favorable it becomes. The reliability is the maintaining quality of a product over some period of time. The reliability function for the Logweighted Power function distribution takes the following formula:

$$
R(y)=1-\frac{y^{\alpha}(1-\alpha \log y)}{\beta^{\alpha}(1-\alpha \log \beta)}, \quad 0<y<\beta<1, \alpha>0
$$

From figure 3 , one can observe that the reliability function decreases, at first, rapidly then decreases slowly for small values of $\alpha$, but it decreases slowly, at first, then it decreases quickly for large values of $\alpha$.

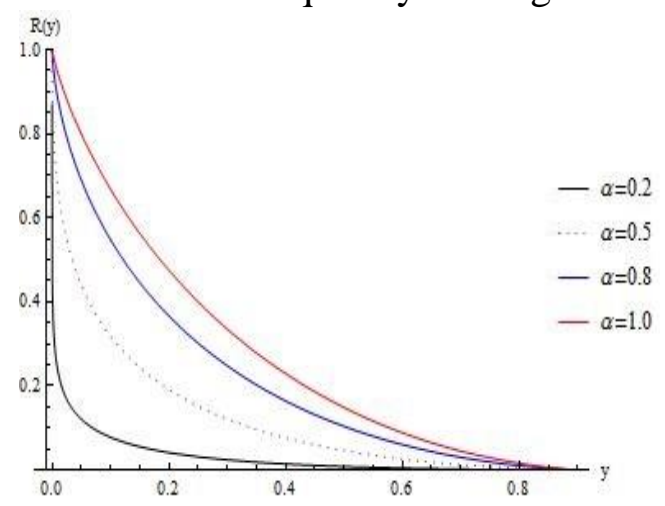

(a) $\alpha \leq 1$

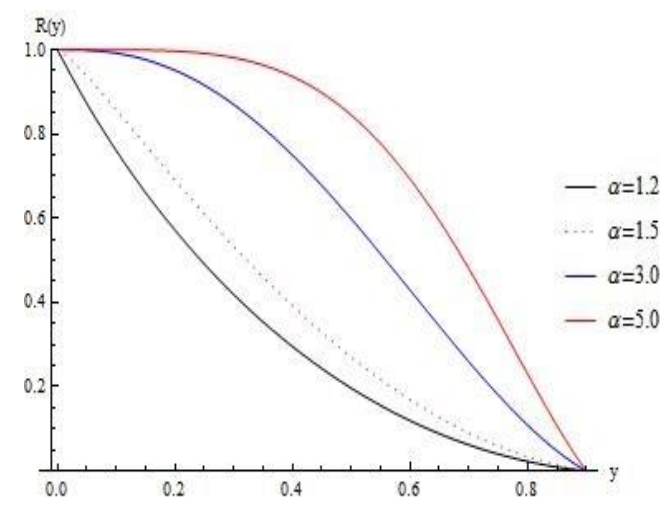

(b) $\alpha>1$

Figure 3. The LWPF Reliability function at $\beta=0.9$ and selected values of $\alpha$

\section{The hazard function:}

One more important function that is used often in reliability is hazard rate function. This can be sometimes used as failure rate depending on the context of use. It gives the instantaneous failure rate of a component. For the Log-weighted Power function distribution, the hazard rate takes the following formula:

$$
h(y)=\frac{\alpha^{2} y^{\alpha-1}(\log y)}{y^{\alpha}(1-\alpha \log y)-\beta^{\alpha}(1-\alpha \log \beta)}, \quad 0<y<\beta<1, \quad \alpha>0
$$

It can be observed from figure 4 that the hazard rate has a bathtub pattern for small values of shape parameter $\alpha$ and as the value of $\alpha$ increases, the hazard rate takes the J-shape. For the bathtub hazard shapes, it is clear that the hazard rate decreases quickly for small values of $Y$ "early failure or burn-in period" and then it begins to increase very slowly "random failure or useful life period" and in the last stage when the value of $Y$ approaches its upper bound, the hazard rate increases quickly "wear-out period". For the J-shape hazard, it is clear that the hazard rate increases slowly for small values of $Y$, then it starts to increase more quickly as the value of $Y$ increases. In addition, the curve of the hazard rate decreases more quickly for large values of scale parameter $\beta$ than its small values. Moreover, the 
curve of the hazard rate moves down as the values of both parameters, $\alpha$ and $\beta$, increase.

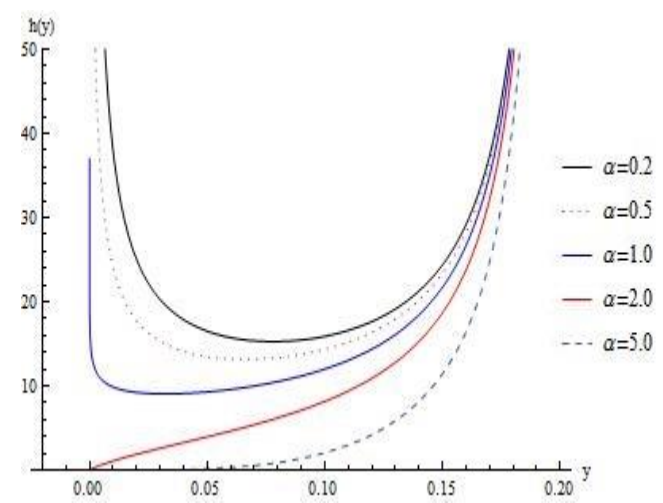

(a) $\beta=0.2$

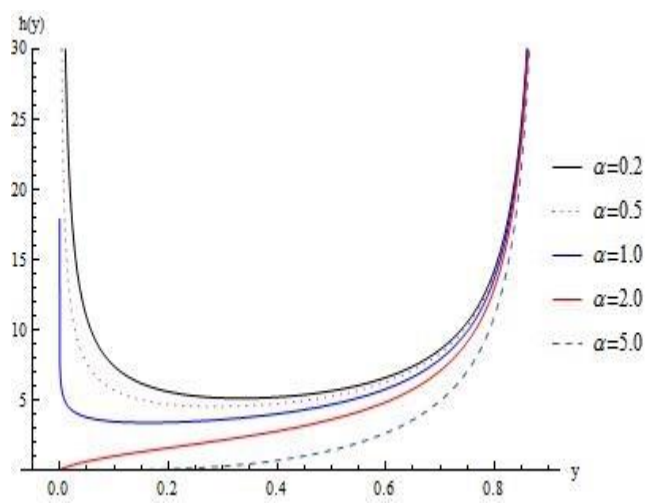

(b) $\beta=0.9$

Figure 4. The hazard function of LWPF distribution at several values of $\alpha$

\section{The reversed hazard rate:}

The reversed hazard rate has different applications in the study of lifetime. The reversed hazard rate of the Log-weighted Power function distribution is given as follows:

$$
\lambda(y)=\frac{\alpha^{2}(\log y)}{y(\alpha \log y-1)}, \quad 0<y<\beta<1, \quad \alpha>0
$$

From the previous formula, it can be observed that the reversed hazard rate is a function in the shape parameter, $\alpha$ and the scale parameter $\beta$ affects only the domain of its function.

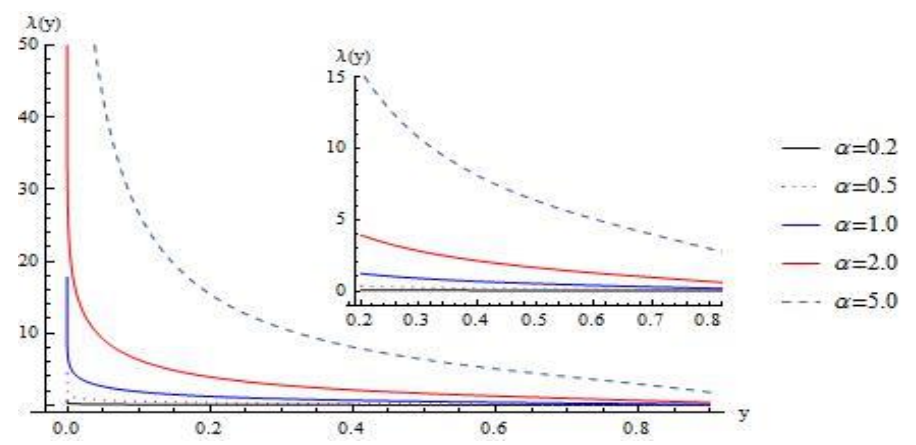

Figure 5. The RHR of the LWPF at $\beta=0.9$ and selected values of $\alpha$

From figure (5), it is clear that the reversed hazard rate is decreasing in Y. Moreover, as the value of the shape parameter $\alpha$ increases, the curve of the reversed hazard rate moves up.

\section{The mean residual life}

The Log-weighted Power function distribution mean residual life is given by:

$$
m(y)=\frac{\beta^{\alpha}(1-\alpha \log \beta)\left((\beta-y)-\frac{\alpha}{(\alpha+1)^{2}}\left(\frac{\alpha+1}{\alpha}\left(\beta^{\alpha+1}(1-\alpha \log \beta)-y^{\alpha+1}(1-\alpha \log y)\right)+\left(\beta^{\alpha+1}-y^{\alpha+1}\right)\right)\right)}{\beta^{\alpha}(1-\alpha \log \beta)-y^{\alpha}(1-\alpha \log y)}
$$




\subsection{The moments}

The rth non-central moment for a random variable follows the Log-weighted Power function is given by:

$$
E\left(y^{r}\right)=\frac{\alpha^{2} \beta^{r}(1-(r+\alpha) \log \beta)}{(r+\alpha)^{2}(1-\alpha \log \beta)}, \quad \alpha>0, \quad 0<\beta<1
$$

Using the previous formula, the expectation, the variance and the coefficient of variation a can be obtained as below:

The expectation

The variance

$$
E(Y)=\frac{\alpha^{2} \beta[1-(\alpha+1) \log \beta]}{(\alpha+1)^{2}[1-\alpha \log \beta]}
$$

$$
V(Z)=\frac{\alpha^{2} \beta^{2}\left[(\alpha+1)^{4}(1-\alpha \log \beta)(1-(\alpha+2) \log \beta)-\alpha^{2}(\alpha+2)^{2}(1-(\alpha+1) \log \beta)\right]}{(\alpha+1)^{4}(\alpha+2)^{2}(1-\alpha \log \beta)^{2}}
$$

The coefficient of variation

$$
C V=\frac{\sqrt{\left[(\alpha+1)^{4}(1-\alpha \log \beta)(1-(\alpha+2) \log \beta)-\alpha^{2}(\alpha+2)^{2}(1-(\alpha+1) \log \beta)\right]}}{\alpha(\alpha+2)[1-(\alpha+1) \log \beta]}
$$

Table 1: Mean and variance of LWPD for some values of $\alpha$ and $\beta$

\begin{tabular}{ccccccccc}
\hline $\boldsymbol{\beta}$ & \multicolumn{2}{c}{0.1} & \multicolumn{2}{c}{0.2} & \multicolumn{2}{c}{0.5} & \multicolumn{2}{c}{0.9} \\
\hline $\boldsymbol{\alpha}$ & Mean & Variance & Mean & Variance & Mean & Variance & Mean & Variance \\
0.5 & 0.02300 & 0.00073 & 0.04204 & 0.00269 & 0.08415 & 0.01321 & 0.11001 & 0.02678 \\
1.0 & 0.04243 & 0.00086 & 0.08084 & 0.00339 & 0.17617 & 0.01948 & 0.24645 & 0.04642 \\
1.5 & 0.05461 & 0.00075 & 0.10594 & 0.00305 & 0.24117 & 0.01896 & 0.35348 & 0.05090 \\
2.0 & 0.06270 & 0.00062 & 0.12280 & 0.00255 & 0.28677 & 0.01657 & 0.43481 & 0.04869 \\
3.0 & 0.07263 & 0.00042 & 0.14357 & 0.00174 & 0.34456 & 0.01180 & 0.54678 & 0.03932 \\
\hline
\end{tabular}

From the previous table, it is obvious that the values of mean are increasing in both $\alpha$ and $\beta$. On the other hand, the value of variance is increasing in $\alpha$, in the interval $\alpha<1$, but its value is decreasing in the interval $\alpha>1$. In addition, if the value of the coefficient of variation is calculated, one can observe that it is decreasing in $\alpha$, but is increasing in $\beta$.

\subsection{Shape Indices}

In order to study the shape of the Log-weighted Power function distribution, a coefficient of skewness and a coefficient of kurtosis are obtained.

\section{Index of Skewness}

To obtain a coefficient of skewness that is independent of the measuring unit, the third order moment is divided by the square root of the variance $\mu_{2}$, raised to the third power. The coefficient of skewness of the Log-weighted Power function distribution is given by:

$$
\sqrt{\beta_{1}}=\frac{\mu_{3}}{\sqrt{\mu_{2}^{3}}}, \quad 0<\beta<1, \quad \alpha>0
$$


where

$\mu_{3}=\mathrm{k}\left[2 \alpha^{2} \beta^{3}\left(2+\alpha \mathrm{A}_{1}+(\alpha+1) \log \beta \mathrm{A}_{2}\right)\right]$,

$\mathrm{k}=\frac{1}{(\alpha+1)^{6}(\alpha+2)^{2}(\alpha+3)^{2}(1-\alpha \log \beta)^{3}}$,

$A_{1}=(14+\alpha(29+\alpha(10-\alpha(5+2 \alpha))))$,

$A_{2}=\left(-6+3 \alpha(-14+\alpha(-29+\alpha(-10+\alpha(5+2 \alpha))))+\alpha(1+\alpha) \log \beta A_{3}\right)$

and

$A_{3}=\left(12+39 \alpha-21 \alpha^{3}-6 \alpha^{4}+(\alpha-1) \alpha(1+\alpha)(2+\alpha)(3+\alpha) \log \beta\right)$

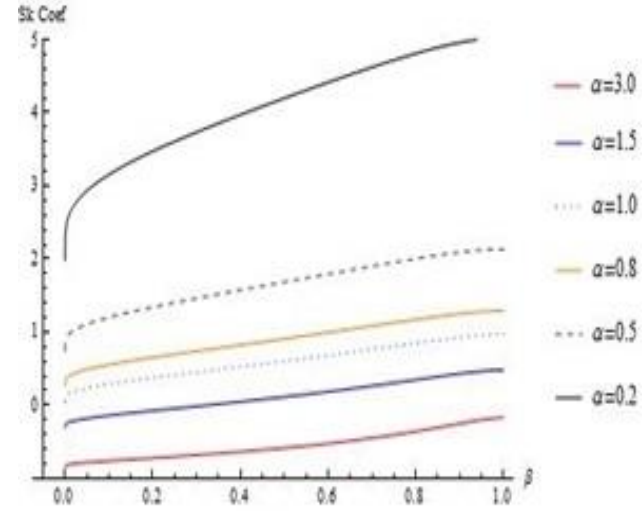

(a)

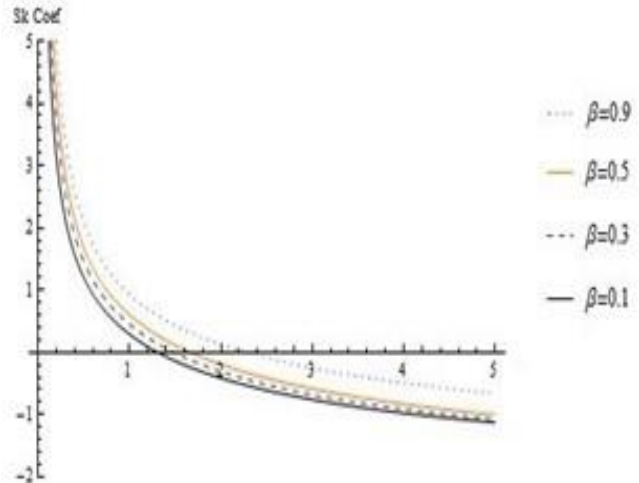

(b)

Figure 6. The coefficient of skewness versus the values of $\alpha$ and $\beta$

From figure 6(a), one can observe that the skewness coefficient increases in $\beta$, keeping the value of $\alpha$ at the same level. However, it can be noticed from figure 6(b) that the value of the coefficient of skewness decreases sharply for the value of $\alpha$, holding the value of $\beta$ constant.

Table 2: Coefficient of Skewness for selected values of $\alpha$ and $\beta$

\begin{tabular}{lrrrrr}
\hline $\boldsymbol{\alpha}$ & $\boldsymbol{\beta}$ & \multicolumn{1}{c}{0.1} & 0.2 & 0.5 & 0.9 \\
\hline $\mathbf{0 . 2}$ & 3.1554 & 3.4730 & 4.1988 & 4.9596 \\
$\mathbf{0 . 5}$ & 1.2031 & 1.3427 & 1.6846 & 2.0929 \\
$\mathbf{1 . 0}$ & 0.2914 & 0.3760 & 0.6036 & 0.9300 \\
$\mathbf{1 . 5}$ & -0.1390 & -0.0741 & 0.1150 & 0.4324 \\
$\mathbf{2 . 0}$ & -0.4133 & 0.3597 & -0.1929 & 0.1285 \\
$\mathbf{3 . 0}$ & -0.7600 & -0.7203 & -0.5834 & -0.2500
\end{tabular}

From table 2, one can observe that as the values of both $\alpha$ and $\beta$ increase, the value of the coefficient of skewness changes from positive sign to negative sign. In other words, the distribution curve transforms from skewed to the right to skewed to the left direction.

\section{Index of Kurtosis}

In order to obtain a coefficient of kurtosis that is independent of the units of 
measurement, the fourth-order moment is divided by the variance of the population, $\sigma^{2}=\mu_{2}$, raised to the second power. For the Log-weighted Power function distribution, the coefficient of kurtosis is given by:

$$
\beta_{2}=\frac{\mu_{4}}{\mu_{2}^{2}}
$$

where

$$
\begin{aligned}
\mu_{4} & =\frac{\mathrm{A}}{\mathrm{B}} \\
\mathrm{A} & =3 \alpha^{2} \beta^{4}\left[\mathrm{~A}_{1}-4 \mathrm{~A}_{2} \log \beta+\alpha^{3}(\alpha+1)^{4}\left(\frac{2 \mathrm{~A}_{3} \log ^{2} \beta}{(\alpha(\alpha+1))^{2}}-\frac{4 \mathrm{~A}_{4} \log ^{3} \beta}{\alpha(\alpha+1)}+\mathrm{A}_{5} \log ^{4} \beta\right)\right] \\
\mathrm{A}_{1} & =12+116 \alpha+423 \alpha^{2}+694 \alpha^{3}+503 \alpha^{4}+288 \alpha^{5}+180 \alpha^{6}+64 \alpha^{7}+8 \alpha^{8}, \\
\mathrm{~A}_{2} & =\left(12+128 \alpha+539 \alpha^{2}+1117 \alpha^{3}+1197 \alpha^{4}+791 \alpha^{5}+468 \alpha^{6}+244 \alpha^{7}+\right. \\
\left.72 \alpha^{8}+8 \alpha^{9}\right) & \\
\mathrm{A}_{3} & =\left(72+460 \alpha+934 \alpha^{2}+649 \alpha^{3}+500 \alpha^{4}+439 \alpha^{5}+172 \alpha^{6}+22 \alpha^{7}\right), \\
\mathrm{A}_{4} & =\left(36+124 \alpha+89 \alpha^{2}+118 \alpha^{3}+123 \alpha^{4}+48 \alpha^{5}+6 \alpha^{6}\right), \\
\mathrm{A}_{5} & =\left(48+28 \alpha+64 \alpha^{2}+71 \alpha^{3}+26 \alpha^{4}+3 \alpha^{5}\right) \text { and } \\
\mathrm{B} & =(\alpha+1)^{8}(\alpha+2)^{2}(\alpha+3)^{2}(\alpha+4)^{2}(\alpha \log (\beta)-1)^{4}
\end{aligned}
$$

\begin{tabular}{|c|c|c|c|c|}
\hline$\beta$ & 0.1 & 0.2 & 0.5 & 0.9 \\
\hline 0.2 & 13.0600 & 15.5995 & 22.5887 & 32.2469 \\
\hline 0.5 & 3.3313 & 3.7645 & 5.0832 & 7.2691 \\
\hline 1.0 & 1.8591 & 1.9272 & 2.2264 & 3.0046 \\
\hline 1.5 & 1.8851 & 1.8630 & 1.8825 & 2.2265 \\
\hline 2.0 & 2.1642 & 2.1040 & 1.9864 & 2.0698 \\
\hline 3.0 & 2.8372 & 2.7498 & 2.5015 & 2.2274 \\
\hline
\end{tabular}

Table 3: Coefficient of Kurtosis for selected values of $\alpha$ and $\beta$

From table (3), one can observe that at a certain value of scale parameter $\beta$, the value of the coefficient of kurtosis decreases in shape parameter $\alpha$ when its values are small, but the value of the coefficient increases when the values of parameter $\alpha$ are large. In addition, For small values of $\alpha$, the coefficient of kurtosis is increasing in parameter $\beta$, but For large values of $\alpha$, the coefficient of kurtosis is decreasing in $\beta$.

\subsection{Order Statistics}

Let $\mathrm{Y}$ be a random variable following the Log-weighted Power function distribution with shape and scale parameters $\alpha$ and $\beta$ respectively. The density function of the $\mathrm{k}^{\text {th }}$ order statistic, $\mathrm{Y}_{(\mathrm{k})}$, from a sample of size $\mathrm{n}$ drawn from a Logweighted Power function distribution has the following form:

$$
f_{k: n}(y)=\frac{n !}{(k-1) !(n-k) !} \frac{\alpha^{2} y^{\alpha-1}(\log y)}{\beta^{\alpha}(\alpha \log \beta-1)}\left(\frac{y^{\alpha}(1-\alpha \log y)}{\beta^{\alpha}(1-\alpha \log \beta)}\right)^{k-1}
$$




$$
\left(1-\frac{y^{\alpha}(1-\alpha \log y)}{\beta^{\alpha}(1-\alpha \log \beta)}\right)^{n-k}, 0<y<\beta<1, \alpha>0
$$

The minimum and the maximum order statistics probability density functions are as follow:

$$
\begin{gathered}
f_{1: n}(y)=\frac{n \alpha^{2} y^{\alpha-1}(\log y)}{\beta^{\alpha}(\alpha \log \beta-1)}\left(1-\frac{y^{\alpha}(1-\alpha \log y)}{\beta^{\alpha}(1-\alpha \log \beta)}\right)^{n-1}, 0<y<\beta<1, \alpha>0 \\
f_{n: n}(y)=\frac{n \alpha^{2} y^{\alpha-1}(\log y)}{\beta^{\alpha}(\alpha \log \beta-1)}\left(\frac{y^{\alpha}(1-\alpha \log y)}{\beta^{\alpha}(1-\alpha \log \beta)}\right)^{n-1}, 0<y<\beta<1, \alpha>0
\end{gathered}
$$

\section{A special case}

If $n=2$, one can obtain the first moment of the first and the second order statistics as follow:

$$
\begin{gathered}
E\left(Y_{1: 2}\right)=\frac{2 \alpha^{4} \beta[(2 \alpha+1) \log \beta((\alpha+1)(2 \alpha+1) \log \beta-4 \alpha-3)+4 \alpha+3]}{(\alpha+1)^{2}(2 \alpha+1)^{3}(\alpha \log \beta-1)^{2}} \\
E\left(Y_{2: 2}\right)=\frac{2 \alpha^{2} \beta[(2 \alpha+1) \log \beta(\alpha(2 \alpha+1) \log \beta-4 \alpha-1)+4 \alpha+1]}{(2 \alpha+1)^{3}(\alpha \log \beta-1)^{2}}
\end{gathered}
$$

\section{Parameter Estimation}

The Log-weighted Power function distribution has two parameters. In order to obtain estimators for these parameters, three different methods are used here, namely, moments method, L-moments method and the maximum likelihood method.

\subsection{The method of moments}

The method of moments is a simple technique based on the idea that the sample moments are "natural" estimators of population moments. Since the distribution has only two parameters, one can equalize the first two moments with the corresponding sample moments. One can obtain the following equations:

$$
\begin{aligned}
& \bar{Y}=\frac{\alpha^{2} \beta(1-(\alpha+1) \log \beta)}{(\alpha+1)^{2}(1-\alpha \log \beta)} \\
& \overline{Y^{2}}=\frac{\alpha^{2} \beta^{2}(1-(\alpha+2) \log \beta)}{(\alpha+2)^{2}(1-\alpha \log \beta)}
\end{aligned}
$$

Solving (23) and (24) simultaneously, we obtain $\widehat{\beta}$ and $\widehat{\alpha}$.

\subsection{L-moments method}

L-moments estimators are more robust to the presence of outliers in the data. Compared with conventional moments, L-moments are less subject to bias in estimation and approximate their asymptotic normal distribution more closely in finite samples. Since there are only two parameters in the current distribution, one can equalize the first two L-moments with the corresponding sample L-moments. 
One can obtain the following equations:

$$
\begin{gathered}
\mathrm{l}_{1}=E(Y) \\
\mathrm{l}_{2}=\frac{1}{2}\left(E\left(Y_{2: 2}\right)-E\left(Y_{1: 2}\right)\right)
\end{gathered}
$$

where

$\mathrm{E}(\mathrm{Y}), \mathrm{E}\left(\mathrm{Y}_{1: 2}\right)$ and $\mathrm{E}\left(\mathrm{Y}_{2: 2}\right)$ are as defined in (13), (21) and (22) respectively.

$\mathrm{l}_{1}=\overline{\mathrm{Y}}$ the sample first L-moment.

$\mathrm{l}_{2}=\frac{1}{2}\left(\begin{array}{l}\mathrm{n} \\ 2\end{array}\right)^{-1} \sum_{\mathrm{i}} \sum_{\mathrm{j}}\left(\mathrm{y}_{\mathrm{i}: \mathrm{n}}-\mathrm{y}_{\mathrm{j}: \mathrm{n}}\right), \mathrm{i}>\mathrm{j}$ the sample second L-moment.

Solving (25) and (26) simultaneously, we obtain $\widehat{\beta}$ and $\widehat{\alpha}$.

\subsection{The maximum likelihood method}

The log-likelihood function of the Log-weighted Power function distribution is given by:

$\log L=2 n \log \alpha-n \alpha \log \beta-n \log (\alpha \log \beta-1)+(\alpha-1) \sum_{i=1}^{n} \log y_{i}+\sum_{i=1}^{n} \log \left(\log y_{i}\right)$

It is clear that the previous function is decreasing in $\beta$, so the value of $\beta$ that maximize this function is the smallest value for $\beta$ which is the largest value of the variable $Y$; i.e. the $n$-th order statistic $Y_{(n)}$, then $\tilde{\beta}=Y_{(n)}$ is the maximum likelihood estimator of $\beta$. Moreover, using the partial derivative of the likelihood function with respect to $\alpha$, one can obtain the following equation:

$$
\frac{2 n}{\alpha}-n \log \beta-\frac{n \log \beta}{(\alpha \log \beta-1)}+\sum_{i=1}^{n} \log y_{i}=0
$$

Substituting $\tilde{\beta}=Y_{(n)}$ in (28), the maximum likelihood estimator, $\widetilde{\alpha}$ of $\alpha$ is obtained.

\section{Simulation Study}

In this section, a study of the behavior of the estimators for the unknown parameters $\alpha$ and $\beta$ is considered. The estimation is made when the two parameters are unknown. Three different estimation methods are used; namely, the maximum likelihood method, the method of moments and the L-moments method. Several combinations of values of the two parameters are assumed. For the shape parameter, $\alpha$, the values $0.5,1.0,2.0,3.0$ and 5.0 were used, where for the scale parameter, $\beta$, the values $0.2,0.5$ and 0.9 were used. The estimated parameters, biases, relative biases, mean squared error (MSE) and scaled root mean squared error (SMSE) were computed. This is done by generating samples from the Log-weighted Power function distribution and considering samples of sizes of 30, 50, 100, 150 and 200. The simulations are based on 10000 replications. The Tables (5-4) through (5-9), in appendix B, present some of the obtained results.

From Table 4, one can observe that all estimates of the shape parameter $\alpha$ and 
almost all estimates of the scale parameter $\beta$ obtained by the L-moments method have the smallest bias among used methods. To the contrary of that, the estimates obtained by the maximum likelihood method are more biased. On the other hand, the values of the mean square error (MSE) of the estimates obtained from the ML method for both parameters $\alpha$ and $\beta$ are smaller than those values obtained from using the method of moments and the L-moments method. In addition, those values of MSE indicate that the estimates obtained from the ML method have higher efficiency than the other two methods. Moreover, almost all of the values of MSE obtained by the L-moments method refer to a higher efficiency than those obtained by the method of moments especially when the values of the shape parameter $\alpha$ are large.

From Table 5, it is clear that the estimates of both parameters, $\alpha$ and $\beta$, obtained by the ML method have the largest values of bias but the smallest values of MSE compared to those estimates obtained by the method of moments and the Lmoments method. On the other hand, the estimates for the shape parameter $\alpha$ obtained by the L-moments method have the minimum bias among the estimates obtained from the estimation methods used. Those estimates are more efficient than those obtain from using the method of moments. Similarly, the same thing occurs for the scale parameter $\beta$ as the values of the shape parameter $\alpha$ go up, but when the values of the shape parameter $\alpha$ are small, the estimates of scale parameter $\beta$ obtained by the method of moments are less bias and more efficient than those obtained by the L-moments method.

From Table 6, the results indicate that the estimates for the shape parameter $\alpha$ obtained by the L-moments method have the minimum bias compared to the other used methods. In addition, the estimates of the scale parameter $\beta$ obtained by the Lmoments method have the minimum bias when the values of the shape parameter $\alpha$ are small, but when the shape parameter $\alpha$ takes larger values, the method of moments gives estimates have less bias compared to the other methods used. On the other hand, the estimates obtained by the ML method have the largest bias compared to the other two methods used, but the values of MSE corresponding to the estimates are the minimum among the methods used. However, the values of MSE corresponding to the estimates of shape parameter $\alpha$ obtained by the Lmoments method are smaller than those obtained by the method of moments, but the opposite is almost true for the scale parameter $\beta$.

In general, the results sited in Table 4 through Table 6 show that the values of bias and MSE are improved as the sample size increases for all estimation methods. Moreover, Tables 7 through 9 display the same comparable results but using the values of relative biases and the scaled root mean square error.

To sum up, it is clear from Table 4 through Table 9 that all the estimates improved in the values of bias, relative bias, MSE and scaled root MSE as the sample size increases. The values of bias and relative bias of estimates obtained by the maximum likelihood method are the largest among the other methods used, but 
on the other hand, those estimates have almost higher efficient compared to both the estimates obtained by the method of moments or the L-moments method according the values of the MSE and scaled root MSE specially for large sample sizes.

\section{Conclusion}

In this paper, the Log-weighted Power function distribution is proposed. A mathematical treatment of the proposed distribution including explicit formulas for the density and hazard functions, moments, order statistics have been provided. The estimation of the parameters has been applied using three estimation methods, namely, the maximum likelihood method and the method of moments and the Lmoments method. A simulation study based on 10000 replications was applied for estimating the distribution parameters by several sample sizes.

\section{References}

[1] Ahmed, A., and Ahmed, A. (2014). Characterization and Estimation of Double Weighted Rayleigh Distribution. Journal of Agriculture and Life Sciences, 1(2), 21-28.

[2] Al-kadim, K. A., and Hantoosh, A. F. (2013). Double Weighted Distribution and Double Weighted Exponential Distribution. Mathematical Theory and Modeling, 3(5), 124-134.

[3] Bashir, S., and Rasul, M. (2018). A New Weighted Rayleigh Distribution: Properties and Applications on Lifetime Time Data. Open Journal of Statistics, 8, 640-650.

[4] Bursa, N. and Kadilar, G. O. (2017). The exponentiated Kumaraswamy power function distribution. Hacettepe University Bulletin of Natural Sciences and Engineering Series Mathematics and Statistics, 46(2), $1-19$.

[5] Chechile, R. A. (2003). Mathematical tools for hazard function analysis. Journal of Mathematical Psychology, 47, 478-494.

[6] Cordeiro, G.M., dos Santos Brito, R. (2012). The beta power distribution. Brazilian Journal of Probability and Statistics, 26(1), 88-112.

[7] Dey, S., Dey, T., and Anis, M. Z. (2015). Weighted Weibull Distribution: Properties and Estimation. Journal of Statistical Theory and Practice, 9, 250-265.

[8] Fisher, R. A. (1934). The Effects of Methods of Ascertainment upon the Estimation of Frequencies. The Annals of Eugenics, 6, 13-25.

[9] Gupta, R., and Kundu, D. (2009). A New Class of Weighted Exponential Distributions. Statistics, 43(6), 621-634. 
[10] ul Haq , M. A, Elgarhy, M., Hashmi, S., Ozel, G. and ul Ain, Q. (2016). Transmuted Weibull Power Function Distribution: its Properties and Applications, Journal of Data Science, 397-418

[11] Hassan, A. S. and Assar, S. M. (2017). Exponentiated Weibull power function distribution applications. Journal of Data Sciences, 15(4), 589-614.

[12] Hosking, J. R. (1990). Analysis and Estimation of Distributions Using Linear Combinations of Order. Journal of the Royal Statistical Society, 52(1), 105-124.

[13] Hussian, M. A. (2013). A Weighted Inverted Exponential Distribution. International Journal of Advanced Statistics and Probability, 1(3), 142-150.

[14] Kersey, J. X., and Oluyede, B. O. (2010). Weighted Inverse Weibull and Beta-Inverse Weibull Distribution. Electronic Theses and Dissertations. 661. https://digitalcommons.georgiasouthern.edu/etd/661.

[15] Levia, V., Sanhueza , A., and Angulo, J. M. (2009). A Length-Biased Version of the Birnbaum-Saunders Distribution with Application in Water Quality. Stoch Environ Res Risk Assess, 23, 299-307.

[16] Mahmoud, A. W., Elbatal, I. I., and Gad, L. S. (2015). About Some New Weighted Distributions. MSc thesis. Institute of Statistical Studies and Research, Cairo University.

[17] Nasiru, S. (2015). Another Weighted Weibull Distribution from Azzalini's Family. European Scientific Journal, 11(9), 134-144.

[18] Oguntunde, P. E., Odetunmibi, O. A., Okagbue, H. I., Babatunde, O. S. and Ugwoke, P. O. (2015). The Kumaraswamy-power distribution: A generalization of the power distribution. International Journal of Mathematical Analysis, 9(13), 637-645.

[19] Okorie, I. E., Akpanta, A. C., Ohakwe, J. and Chikezie, D. C. (2017). The modified power function distribution. Cogent Mathematics, 4, 1-19. https://doi.org/10.1080/23311835. 2017.1319592 .

[20] Patil, G. P. (2002). Weighted Distributions. (El-Shaarawi, A. and Piegorsch, W. W., Eds.) in Encyclopedia of Environmetrics, 4, pp. 2369-2377.

[21] Rao, C. R. (1965). On Discrete Distributions Arising out of Methods of Ascertainment. Sankhya: The Indian Journal of Statistics, Series A (1961-2002), 27(2/4), 311-324.

[22] Saghir, A., and Saleem, M. (2016). Double Weighted Weibull Distribution Properties and Application. Mathematical Theory and Modeling, 6, 28-46.

[23] Seenoi, P., Supapakorn, T., and Bodhisuwan, W. (2014). The Length-Biased Exponentiated Inverted Weibull Distribution. International Journal of Pure and Applied Mathematics, 92(2), 191-206. 
[24] Shaban, S. A., and Boudrissa, N. A. (2007). The Weibull Length Biased DistributionProperties and Estimation, InterStat, January 2007.

[25] Shi, X., Oluyede, B., and Pararai, M. (2012). Theoretical Properties of Weighted Generalized Rayleigh and Related Distributions. Theoretical Mathematics and Applications, 2(2), 45-62.

[26] Tahir, M., Alizadeh, M., Mansoor, M., Cordeiro, G. M. and Zubair, M. (2016 a). The Weibull-power function distribution with applications. Hacettepe University Bulletin of Natural Sciences and Engineering Series Mathematics and Statistics, 45(1), 245 - 265. 
272 A Log-Weighted Power Function Distribution and Its Statistical Properties

\section{Appendix 1: Tables}

Table 4: Biases and MSEs of $\widehat{\alpha}$ and $\widehat{\beta}$ using different estimation methods at $\beta=0.2$ and several values of $\alpha$

\begin{tabular}{|c|c|c|c|c|c|c|c|c|c|c|c|c|c|}
\hline \multirow[b]{3}{*}{$\alpha$} & \multirow[b]{3}{*}{$n$} & \multicolumn{4}{|c|}{ Maximum likelihood } & \multicolumn{4}{|c|}{ L-moments } & \multicolumn{4}{|c|}{ Moments } \\
\hline & & \multicolumn{2}{|c|}{$\widehat{\alpha}$} & \multicolumn{2}{|c|}{$\widehat{\boldsymbol{\beta}}$} & \multicolumn{2}{|c|}{$\widehat{\alpha}$} & \multicolumn{2}{|c|}{$\widehat{\boldsymbol{\beta}}$} & \multicolumn{2}{|c|}{$\widehat{\alpha}$} & \multicolumn{2}{|c|}{$\widehat{\boldsymbol{\beta}}$} \\
\hline & & Bias & MSE & Bias & MSE & Bias & MSE & Bias & MSE & Bias & MSE & Bias & MSE \\
\hline \multirow{5}{*}{0.5} & 30 & 0.02898 & 0.00631 & -0.02573 & 0.00115 & -0.00570 & 0.00753 & 0.00683 & 0.00173 & 0.01410 & 0.00884 & -0.00530 & 0.00100 \\
\hline & 50 & 0.01940 & 0.00340 & -0.01619 & 0.00050 & -0.00325 & 0.00434 & 0.00429 & 0.00092 & 0.00931 & 0.00497 & -0.00302 & 0.00059 \\
\hline & 100 & 0.01030 & 0.00150 & -0.00840 & 0.00010 & -0.00210 & 0.00211 & 0.00207 & 0.00044 & 0.00441 & 0.00236 & -0.00163 & 0.00031 \\
\hline & 150 & 0.00890 & 0.00100 & -0.00580 & 0.00010 & -0.00115 & 0.00136 & 0.00121 & 0.00028 & 0.00315 & 0.00152 & -0.00121 & 0.00020 \\
\hline & 200 & 0.00780 & 0.00070 & -0.00430 & 0.00000 & -0.00088 & 0.00102 & 0.00085 & 0.00020 & 0.00221 & 0.00114 & -0.00089 & 0.00015 \\
\hline \multirow{5}{*}{1.0} & 30 & 0.05410 & 0.02790 & -0.01020 & 0.00020 & 0.00370 & 0.02770 & 0.00220 & 0.00040 & 0.03200 & 0.02920 & -0.00280 & 0.00030 \\
\hline & 50 & 0.03160 & 0.01490 & -0.00630 & 0.00010 & 0.00080 & 0.01600 & 0.00140 & 0.00020 & 0.01810 & 0.01670 & -0.00160 & 0.00020 \\
\hline & 100 & 0.01621 & 0.00670 & -0.00316 & 0.00002 & 0.00132 & 0.00756 & 0.00067 & 0.00011 & 0.00995 & 0.00778 & -0.00081 & 0.00010 \\
\hline & 150 & 0.00948 & 0.00429 & -0.00210 & 0.00001 & -0.00034 & 0.00499 & 0.00037 & 0.00007 & 0.00529 & 0.00509 & -0.00060 & 0.00006 \\
\hline & 200 & 0.00812 & 0.00324 & -0.00160 & 0.00000 & 0.00050 & 0.00385 & 0.00052 & 0.00005 & 0.00485 & 0.00392 & -0.00023 & 0.00005 \\
\hline \multirow{5}{*}{2.0} & 30 & 0.11713 & 0.13167 & -0.00420 & 0.00003 & 0.03072 & 0.12955 & 0.00092 & 0.00011 & 0.04938 & 0.11967 & -0.00031 & 0.00013 \\
\hline & 50 & 0.06762 & 0.06887 & -0.00257 & 0.00001 & 0.01704 & 0.07177 & 0.00043 & 0.00006 & 0.02905 & 0.06570 & -0.00036 & 0.00006 \\
\hline & 100 & 0.03522 & 0.03113 & -0.00129 & 0.00000 & 0.01015 & 0.03394 & 0.00020 & 0.00003 & 0.01749 & 0.03221 & -0.00024 & 0.00003 \\
\hline & 150 & 0.02260 & 0.02052 & -0.00088 & 0.00000 & 0.00592 & 0.02330 & 0.00015 & 0.00002 & 0.01125 & 0.02235 & -0.00016 & 0.00002 \\
\hline & 200 & 0.01619 & 0.01479 & -0.00066 & 0.00000 & 0.00394 & 0.01669 & 0.00009 & 0.00001 & 0.00819 & 0.01608 & -0.00016 & 0.00002 \\
\hline \multirow{5}{*}{3.0} & 30 & 0.17875 & 0.32179 & -0.00261 & 0.00001 & 0.05998 & 0.32464 & 0.00005 & 0.00005 & 0.04397 & 0.38027 & 0.00457 & 0.00193 \\
\hline & 50 & 0.10508 & 0.16540 & -0.00161 & 0.00001 & 0.03642 & 0.17859 & 0.00020 & 0.00003 & 0.05795 & 0.21469 & 0.00094 & 0.00059 \\
\hline & 100 & 0.05081 & 0.07634 & -0.00079 & 0.00000 & 0.01604 & 0.08634 & 0.00016 & 0.00001 & 0.03835 & 0.09406 & -0.00025 & 0.00003 \\
\hline & 150 & 0.03775 & 0.05051 & -0.00053 & 0.00000 & 0.01437 & 0.05785 & 0.00011 & 0.00001 & 0.03067 & 0.06234 & -0.00022 & 0.00001 \\
\hline & 200 & 0.02867 & 0.03558 & -0.00041 & 0.00000 & 0.01092 & 0.04085 & 0.00007 & 0.00001 & 0.02362 & 0.04358 & -0.00019 & 0.00001 \\
\hline \multirow{5}{*}{5.0} & 30 & 0.31594 & 0.96697 & -0.00147 & 0.00000 & 0.14078 & 1.01537 & 0.00020 & 0.00002 & -0.31434 & 1.94259 & 0.00859 & 0.00269 \\
\hline & 50 & 0.18112 & 0.50375 & -0.00090 & 0.00000 & 0.08027 & 0.56363 & 0.00008 & 0.00001 & -0.46022 & 1.36860 & 0.00697 & 0.00080 \\
\hline & 100 & 0.08398 & 0.23062 & -0.00045 & 0.00000 & 0.03769 & 0.26965 & 0.00002 & 0.00001 & -0.55584 & 0.95675 & 0.00636 & 0.00009 \\
\hline & 150 & 0.05966 & 0.14793 & -0.00030 & 0.00000 & 0.02798 & 0.17633 & 0.00003 & 0.00000 & -0.53552 & 0.78090 & 0.00594 & 0.00008 \\
\hline & 200 & 0.04112 & 0.10749 & -0.00023 & 0.00000 & 0.01574 & 0.12785 & 0.00002 & 0.00000 & -0.50070 & 0.67006 & 0.00540 & 0.00007 \\
\hline
\end{tabular}


Table 5: Biases and MSEs of $\widehat{\alpha}$ and $\widehat{\beta}$ using different estimation methods at $\beta=0.5$ and several values of $\alpha$

\begin{tabular}{|c|c|c|c|c|c|c|c|c|c|c|c|c|c|}
\hline \multirow[b]{3}{*}{$\alpha$} & \multirow[b]{3}{*}{$\boldsymbol{n}$} & \multicolumn{4}{|c|}{ Maximum likelihood } & \multicolumn{4}{|c|}{ L-moments } & \multicolumn{4}{|c|}{ Moments } \\
\hline & & \multicolumn{2}{|c|}{$\widehat{\alpha}$} & \multicolumn{2}{|c|}{$\widehat{\boldsymbol{\beta}}$} & \multicolumn{2}{|c|}{$\widehat{\alpha}$} & \multicolumn{2}{|c|}{$\widehat{\boldsymbol{\beta}}$} & \multicolumn{2}{|c|}{$\widehat{\alpha}$} & \multicolumn{2}{|c|}{$\widehat{\boldsymbol{\beta}}$} \\
\hline & & Bias & MSE & Bias & MSE & Bias & MSE & Bias & MSE & Bias & MSE & Bias & MSE \\
\hline \multirow{3}{*}{0.5} & 50 & 0.01471 & 0.00303 & -0.06112 & 0.00632 & -0.00270 & 0.00396 & 0.02578 & 0.01593 & 0.01053 & 0.00464 & -0.00947 & 0.00764 \\
\hline & 100 & 0.00745 & 0.00143 & -0.03415 & 0.00209 & -0.00180 & 0.00200 & 0.01039 & 0.00636 & 0.00453 & 0.00232 & -0.00514 & 0.00371 \\
\hline & 150 & 0.00536 & 0.00089 & -0.02365 & 0.00103 & -0.00086 & 0.00130 & 0.00663 & 0.00407 & 0.00335 & 0.00150 & -0.00356 & 0.00249 \\
\hline \multirow{5}{*}{1.0} & 30 & 0.05311 & 0.02486 & -0.03547 & 0.00225 & 0.00536 & 0.02412 & 0.01047 & 0.00504 & 0.03337 & 0.02609 & -0.00784 & 0.00350 \\
\hline & 50 & 0.02809 & 0.01293 & -0.02245 & 0.00094 & -0.00123 & 0.01387 & 0.00564 & 0.00271 & 0.01520 & 0.01448 & -0.00494 & 0.00203 \\
\hline & 100 & 0.01344 & 0.00588 & -0.01178 & 0.00036 & -0.00095 & 0.00675 & 0.00307 & 0.00130 & 0.00738 & 0.00697 & -0.00221 & 0.00100 \\
\hline & 150 & 0.00999 & 0.00401 & -0.00789 & 0.00012 & 0.00100 & 0.00468 & 0.00133 & 0.00083 & 0.00629 & 0.00482 & -0.00200 & 0.00066 \\
\hline & 200 & 0.00667 & 0.00292 & -0.00591 & 0.00007 & -0.00052 & 0.00344 & 0.00147 & 0.00063 & 0.00351 & 0.00354 & -0.00107 & 0.00050 \\
\hline \multirow{4}{*}{2.0} & 30 & 0.10877 & 0.11118 & -0.01369 & 0.00036 & 0.02503 & 0.10906 & 0.00318 & 0.00102 & 0.07420 & 0.11857 & -0.00433 & 0.00099 \\
\hline & 50 & 0.05935 & 0.05797 & -0.00826 & 0.00013 & 0.01090 & 0.06179 & 0.00193 & 0.00059 & 0.03964 & 0.06503 & -0.00247 & 0.00059 \\
\hline & 150 & 0.02054 & 0.01709 & -0.00283 & 0.00002 & 0.00547 & 0.01960 & 0.00019 & 0.00018 & 0.01502 & 0.01995 & -0.00127 & 0.00019 \\
\hline & 200 & 0.01374 & 0.01215 & -0.00216 & 0.00001 & 0.00085 & 0.01390 & 0.00046 & 0.00014 & 0.00799 & 0.01403 & -0.00064 & 0.00014 \\
\hline \multirow{5}{*}{3.0} & 30 & 0.16199 & 0.26854 & -0.00793 & 0.00012 & 0.05160 & 0.27444 & 0.00153 & 0.00043 & 0.12974 & 0.31208 & -0.00336 & 0.00050 \\
\hline & 50 & 0.10324 & 0.14142 & -0.00497 & 0.00005 & 0.04021 & 0.15263 & 0.00048 & 0.00025 & 0.08701 & 0.17096 & -0.00242 & 0.00030 \\
\hline & 100 & 0.04571 & 0.06416 & -0.00243 & 0.00001 & 0.01481 & 0.07322 & 0.00027 & 0.00012 & 0.03818 & 0.07869 & -0.00121 & 0.00015 \\
\hline & 150 & 0.03304 & 0.04164 & -0.00165 & 0.00001 & 0.01147 & 0.04750 & 0.00024 & 0.00008 & 0.02675 & 0.05038 & -0.00074 & 0.00010 \\
\hline & 200 & 0.02618 & 0.03132 & -0.00125 & 0.00000 & 0.01002 & 0.03601 & 0.00017 & 0.00006 & 0.02150 & 0.03809 & -0.00056 & 0.00007 \\
\hline \multirow{5}{*}{5.0} & 30 & 0.29947 & 0.85881 & -0.00427 & 0.00004 & 0.13156 & 0.89882 & 0.00055 & 0.00014 & 0.21442 & 0.95435 & -0.00144 & 0.00022 \\
\hline & 50 & 0.18283 & 0.45498 & -0.00259 & 0.00001 & 0.08470 & 0.49789 & 0.00027 & 0.00008 & 0.13955 & 0.52169 & -0.00104 & 0.00011 \\
\hline & 100 & 0.08768 & 0.20090 & -0.00130 & 0.00000 & 0.04100 & 0.23274 & 0.00010 & 0.00004 & 0.07632 & 0.25244 & -0.00068 & 0.00006 \\
\hline & 150 & 0.05876 & 0.12433 & -0.00086 & 0.00000 & 0.02924 & 0.14925 & 0.00003 & 0.00003 & 0.05709 & 0.16745 & -0.00057 & 0.00004 \\
\hline & 200 & 0.04083 & 0.09315 & -0.00064 & 0.00000 & 0.01781 & 0.11107 & 0.00007 & 0.00002 & 0.03850 & 0.12493 & -0.00037 & 0.00003 \\
\hline
\end{tabular}


Table 6: Biases and MSEs of $\widehat{\alpha}$ and $\widehat{\beta}$ using different estimation methods at $\beta=0.9$ and several values of $\alpha$ 
Table 7: Relative Biases and scaled root MSEs of $\widehat{\alpha}$ and $\widehat{\beta}$ using different estimation methods at $\beta=0.2$ and several values of $\alpha$

\begin{tabular}{|c|c|c|c|c|c|c|c|c|c|c|c|c|c|}
\hline \multirow[b]{4}{*}{$\alpha$} & \multirow[b]{4}{*}{$\boldsymbol{n}$} & \multicolumn{12}{|c|}{$\beta=0.2$} \\
\hline & & \multicolumn{4}{|c|}{ Maximum likelihood } & \multicolumn{4}{|c|}{ L-moments } & \multicolumn{4}{|c|}{ Moments } \\
\hline & & \multicolumn{2}{|c|}{$\widehat{\alpha}$} & \multicolumn{2}{|c|}{$\widehat{\boldsymbol{\beta}}$} & \multicolumn{2}{|c|}{$\widehat{\alpha}$} & \multicolumn{2}{|c|}{$\widehat{\boldsymbol{\beta}}$} & \multicolumn{2}{|c|}{$\widehat{\alpha}$} & \multicolumn{2}{|c|}{$\widehat{\boldsymbol{\beta}}$} \\
\hline & & R.Bias & SMSE & R.Bias & SMSE & R.Bias & SMSE & R.Bias & SMSE & R.Bias & SMSE & R.Bias & SMSE \\
\hline \multirow{5}{*}{0.5} & 30 & 0.05800 & 0.15875 & -0.12850 & 0.16583 & -0.01140 & 0.17321 & 0.03400 & 0.20616 & 0.02820 & 0.18762 & -0.02650 & 0.15811 \\
\hline & 50 & 0.03880 & 0.11662 & -0.08100 & 0.11180 & -0.00640 & 0.13115 & 0.02150 & 0.15000 & 0.01860 & 0.14142 & -0.01500 & 0.12247 \\
\hline & 100 & 0.02060 & 0.07746 & -0.04200 & 0.05000 & -0.00420 & 0.09165 & 0.01050 & 0.10000 & 0.00880 & 0.09798 & -0.00800 & 0.08660 \\
\hline & 150 & 0.01780 & 0.06325 & -0.02900 & 0.05000 & -0.00220 & 0.07483 & 0.00600 & 0.08660 & 0.00640 & 0.07746 & -0.00600 & 0.07071 \\
\hline & 200 & 0.01560 & 0.05292 & -0.02150 & 0.00000 & -0.00180 & 0.06325 & 0.00450 & 0.07071 & 0.00440 & 0.06633 & -0.00450 & 0.05000 \\
\hline \multirow{5}{*}{1.0} & 30 & 0.05410 & 0.16703 & -0.05100 & 0.07071 & 0.00370 & 0.16643 & 0.01100 & 0.10000 & 0.03200 & 0.17088 & -0.01400 & 0.08660 \\
\hline & 50 & 0.03160 & 0.12207 & -0.03150 & 0.05000 & 0.00080 & 0.12649 & 0.00700 & 0.07071 & 0.01810 & 0.12923 & -0.00800 & 0.07071 \\
\hline & 100 & 0.01621 & 0.08185 & -0.01582 & 0.02202 & 0.00132 & 0.08697 & 0.00337 & 0.05299 & 0.00995 & 0.08823 & -0.00406 & 0.04914 \\
\hline & 150 & 0.00948 & 0.06550 & -0.01050 & 0.01472 & -0.00034 & 0.07065 & 0.00187 & 0.04319 & 0.00529 & 0.07137 & -0.00298 & 0.04025 \\
\hline & 200 & 0.00812 & 0.05696 & -0.00802 & 0.01002 & 0.00050 & 0.06206 & 0.00258 & 0.03701 & 0.00485 & 0.06264 & -0.00117 & 0.03442 \\
\hline \multirow{4}{*}{2.0} & 30 & 0.05856 & 0.18143 & -0.02100 & 0.02941 & 0.01536 & 0.17996 & 0.00459 & 0.05158 & 0.02469 & 0.17296 & -0.00154 & 0.05707 \\
\hline & 100 & 0.01761 & 0.08822 & -0.00647 & 0.00902 & 0.00508 & 0.09211 & 0.00099 & 0.02683 & 0.00875 & 0.08974 & -0.00120 & 0.02646 \\
\hline & 150 & 0.01130 & 0.07162 & -0.00438 & 0.00612 & 0.00296 & 0.07633 & 0.00077 & 0.02208 & 0.00563 & 0.07476 & -0.00080 & 0.02230 \\
\hline & 200 & 0.00809 & 0.06080 & -0.00329 & 0.00461 & 0.00197 & 0.06459 & 0.00044 & 0.01917 & 0.00409 & 0.06341 & -0.00078 & 0.01962 \\
\hline \multirow{5}{*}{3.0} & 30 & 0.05958 & 0.18909 & -0.01303 & 0.01844 & 0.01999 & 0.18993 & 0.00025 & 0.03464 & 0.01466 & 0.20555 & 0.02286 & 0.21949 \\
\hline & 50 & 0.03503 & 0.13557 & -0.00805 & 0.01118 & 0.01214 & 0.14086 & 0.00101 & 0.02683 & 0.01932 & 0.15445 & 0.00470 & 0.12124 \\
\hline & 100 & 0.01694 & 0.09210 & -0.00396 & 0.00557 & 0.00535 & 0.09795 & 0.00078 & 0.01823 & 0.01278 & 0.10223 & -0.00127 & 0.02859 \\
\hline & 150 & 0.01258 & 0.07492 & -0.00267 & 0.00376 & 0.00479 & 0.08018 & 0.00054 & 0.01513 & 0.01022 & 0.08322 & -0.00108 & 0.01737 \\
\hline & 200 & 0.00956 & 0.06288 & -0.00203 & 0.00274 & 0.00364 & 0.06737 & 0.00034 & 0.01294 & 0.00787 & 0.06959 & -0.00094 & 0.01499 \\
\hline \multirow{5}{*}{5.0} & 30 & 0.06319 & 0.19667 & -0.00737 & 0.01038 & 0.02816 & 0.20153 & 0.00099 & 0.02121 & -0.06287 & 0.27875 & 0.04296 & 0.25928 \\
\hline & 50 & 0.03622 & 0.14195 & -0.00451 & 0.00612 & 0.01605 & 0.15015 & 0.00041 & 0.01612 & -0.09204 & 0.23397 & 0.03485 & 0.14107 \\
\hline & 100 & 0.01680 & 0.09605 & -0.00226 & 0.00316 & 0.00754 & 0.10386 & 0.00008 & 0.01140 & -0.11117 & 0.19563 & 0.03182 & 0.04743 \\
\hline & 150 & 0.01193 & 0.07692 & -0.00149 & 0.00206 & 0.00560 & 0.08398 & 0.00014 & 0.00922 & -0.10710 & 0.17674 & 0.02969 & 0.04536 \\
\hline & 200 & 0.00822 & 0.06557 & -0.00114 & 0.00161 & 0.00315 & 0.07151 & 0.00011 & 0.00250 & -0.10014 & 0.16371 & 0.02700 & 0.04292 \\
\hline
\end{tabular}


Rasha Mohamed Mandouh, Mahmoud Abdel-Ghaffar Mohamed

277

Table 8: Relative Biases and scaled root MSEs of $\widehat{\alpha}$ and $\hat{\beta}$ using different estimation methods at $\beta=0.5$ and several values of $\alpha$

\begin{tabular}{|c|c|c|c|c|c|c|c|c|c|c|c|c|c|}
\hline \multirow{4}{*}{$\alpha$} & \multirow{4}{*}{$\begin{array}{l} \\
n\end{array}$} & \multicolumn{12}{|c|}{$\beta=0.5$} \\
\hline & & \multicolumn{4}{|c|}{ Maximum likelihood } & \multicolumn{4}{|c|}{ L-moments } & \multicolumn{4}{|c|}{ Moments } \\
\hline & & \multicolumn{2}{|c|}{$\widehat{\alpha}$} & \multicolumn{2}{|c|}{$\widehat{\boldsymbol{\beta}}$} & \multicolumn{2}{|c|}{$\widehat{\alpha}$} & \multicolumn{2}{|c|}{$\widehat{\boldsymbol{\beta}}$} & \multicolumn{2}{|c|}{$\widehat{\alpha}$} & \multicolumn{2}{|c|}{$\widehat{\boldsymbol{\beta}}$} \\
\hline & & R.Bias & SMSE & R.Bias & SMSE & R.Bias & SMSE & R.Bias & SMSE & R.Bias & SMSE & R.Bias & SMSE \\
\hline \multirow{5}{*}{0.5} & 30 & 0.05122 & 0.15211 & -0.18119 & 0.22839 & -0.00661 & 0.16590 & 0.09735 & 0.36684 & 0.03678 & 0.18055 & -0.02858 & 0.23765 \\
\hline & 50 & 0.02942 & 0.11009 & -0.12225 & 0.15897 & -0.00541 & 0.12584 & 0.05155 & 0.25244 & 0.02106 & 0.13623 & -0.01894 & 0.17487 \\
\hline & 100 & 0.01490 & 0.07567 & -0.06829 & 0.09147 & -0.00359 & 0.08938 & 0.02077 & 0.15949 & 0.00906 & 0.09636 & -0.01027 & 0.12189 \\
\hline & 150 & 0.01072 & 0.05978 & -0.04730 & 0.06418 & -0.00171 & 0.07201 & 0.01327 & 0.12762 & 0.00670 & 0.07736 & -0.00711 & 0.09987 \\
\hline & 200 & 0.00813 & 0.05166 & -0.03627 & 0.04930 & -0.00214 & 0.06136 & 0.01334 & 0.10987 & 0.00452 & 0.06576 & -0.00270 & 0.08587 \\
\hline \multirow{5}{*}{1.0} & 30 & 0.05311 & 0.15766 & -0.07093 & 0.09485 & 0.00536 & 0.15530 & 0.02093 & 0.14200 & 0.03337 & 0.16151 & -0.01567 & 0.11826 \\
\hline & 50 & 0.02809 & 0.11371 & -0.04490 & 0.06126 & -0.00123 & 0.11777 & 0.01127 & 0.10403 & 0.01520 & 0.12033 & -0.00988 & 0.09007 \\
\hline & 100 & 0.01344 & 0.07667 & -0.02356 & 0.03792 & -0.00095 & 0.08216 & 0.00614 & 0.07203 & 0.00738 & 0.08351 & -0.00442 & 0.06339 \\
\hline & 150 & 0.00999 & 0.06332 & -0.01579 & 0.02175 & 0.00100 & 0.06842 & 0.00266 & 0.05766 & 0.00629 & 0.06944 & -0.00400 & 0.05120 \\
\hline & 200 & 0.00667 & 0.05404 & -0.01181 & 0.01660 & -0.00052 & 0.05864 & 0.00295 & 0.05010 & 0.00351 & 0.05947 & -0.00214 & 0.04451 \\
\hline \multirow{4}{*}{2.0} & 30 & 0.05439 & 0.16672 & -0.02739 & 0.03792 & 0.01252 & 0.16512 & 0.00636 & 0.06381 & 0.03710 & 0.17217 & -0.00865 & 0.06285 \\
\hline & 100 & 0.01635 & 0.08152 & -0.00854 & 0.01202 & 0.00439 & 0.08548 & 0.00113 & 0.03332 & 0.01158 & 0.08672 & -0.00326 & 0.03387 \\
\hline & 150 & 0.01027 & 0.06537 & -0.00566 & 0.00794 & 0.00274 & 0.07000 & 0.00038 & 0.02698 & 0.00751 & 0.07062 & -0.00255 & 0.02748 \\
\hline & 200 & 0.00687 & 0.05510 & -0.00433 & 0.00614 & 0.00042 & 0.05894 & 0.00091 & 0.02350 & 0.00400 & 0.05923 & -0.00129 & 0.02395 \\
\hline \multirow{5}{*}{3.0} & 30 & 0.05400 & 0.17274 & -0.01586 & 0.02219 & 0.01720 & 0.17462 & 0.00305 & 0.04148 & 0.04325 & 0.18621 & -0.00671 & 0.04491 \\
\hline & 50 & 0.03441 & 0.12535 & -0.00994 & 0.01405 & 0.01340 & 0.13023 & 0.00095 & 0.03150 & 0.02900 & 0.13782 & -0.00485 & 0.03480 \\
\hline & 100 & 0.01524 & 0.08443 & -0.00486 & 0.00680 & 0.00494 & 0.09020 & 0.00055 & 0.02192 & 0.01273 & 0.09351 & -0.00242 & 0.02427 \\
\hline & 150 & 0.01101 & 0.06802 & -0.00329 & 0.00464 & 0.00382 & 0.07265 & 0.00047 & 0.01774 & 0.00892 & 0.07482 & -0.00147 & 0.01971 \\
\hline & 200 & 0.00873 & 0.05899 & -0.00250 & 0.00355 & 0.00334 & 0.06325 & 0.00034 & 0.01516 & 0.00717 & 0.06505 & -0.00112 & 0.01687 \\
\hline \multirow{5}{*}{5.0} & 30 & 0.05989 & 0.18534 & -0.00853 & 0.01194 & 0.02631 & 0.18961 & 0.00111 & 0.02401 & 0.04288 & 0.19538 & -0.00289 & 0.02989 \\
\hline & 50 & 0.03657 & 0.13490 & -0.00518 & 0.00734 & 0.01694 & 0.14112 & 0.00055 & 0.01829 & 0.02791 & 0.14446 & -0.00208 & 0.02097 \\
\hline & 100 & 0.01754 & 0.08964 & -0.00259 & 0.00364 & 0.00820 & 0.09649 & 0.00021 & 0.01270 & 0.01526 & 0.10049 & -0.00136 & 0.01531 \\
\hline & 150 & 0.01175 & 0.07052 & -0.00173 & 0.00242 & 0.00585 & 0.07727 & 0.00005 & 0.01029 & 0.01142 & 0.08184 & -0.00114 & 0.01266 \\
\hline & 200 & 0.00817 & 0.06104 & -0.00128 & 0.00182 & 0.00356 & 0.06666 & 0.00013 & 0.00895 & 0.00770 & 0.07069 & -0.00073 & 0.01108 \\
\hline
\end{tabular}


Table 9: Relative Biases and scaled root MSEs of $\widehat{\alpha}$ and $\widehat{\beta}$ using different estimation methods at $\beta=0.9$ and several values of $\alpha$

\begin{tabular}{|c|c|c|c|c|c|c|c|c|c|c|c|c|c|}
\hline \multirow{4}{*}{$\alpha$} & \multirow{4}{*}{$n$} & \multicolumn{12}{|c|}{$\beta=0.9$} \\
\hline & & \multicolumn{4}{|c|}{ Maximum likelihood } & \multicolumn{4}{|c|}{ L-moments } & \multicolumn{4}{|c|}{ Moments } \\
\hline & & \multicolumn{2}{|c|}{$\widehat{\alpha}$} & \multicolumn{2}{|c|}{$\widehat{\boldsymbol{\beta}}$} & \multicolumn{2}{|c|}{$\widehat{\alpha}$} & \multicolumn{2}{|c|}{$\widehat{\boldsymbol{\beta}}$} & \multicolumn{2}{|c|}{$\widehat{\alpha}$} & \multicolumn{2}{|c|}{$\widehat{\boldsymbol{\beta}}$} \\
\hline & & R.Bias & SMSE & R.Bias & SMSE & R.Bias & SMSE & R.Bias & SMSE & R.Bias & SMSE & R.Bias & SMSE \\
\hline \multirow{3}{*}{0.5} & 50 & 0.03005 & 0.10981 & -0.24151 & 0.27899 & 0.00506 & 0.13832 & -0.06998 & 0.22496 & 0.01239 & 0.13700 & -0.08746 & 0.23365 \\
\hline & 100 & 0.01512 & 0.07353 & -0.16406 & 0.19435 & 0.00957 & 0.09734 & -0.04848 & 0.18642 & 0.00658 & 0.09740 & -0.05282 & 0.18340 \\
\hline & 150 & 0.01070 & 0.05935 & -0.12730 & 0.15344 & 0.01165 & 0.07895 & -0.03667 & 0.16587 & 0.00724 & 0.07967 & -0.03696 & 0.16004 \\
\hline \multirow{5}{*}{1.0} & 30 & 0.04918 & 0.15011 & -0.15601 & 0.18668 & 0.00968 & 0.14673 & -0.03079 & 0.16729 & 0.02600 & 0.15086 & -0.05320 & 0.17378 \\
\hline & $\mathbf{5 0}$ & 0.03043 & 0.10988 & -0.11128 & 0.13588 & 0.00618 & 0.11258 & -0.01795 & 0.14350 & 0.01584 & 0.11554 & -0.03193 & 0.14303 \\
\hline & 100 & 0.01401 & 0.07392 & -0.06814 & 0.08541 & 0.00198 & 0.07938 & -0.00355 & 0.11858 & 0.00795 & 0.07788 & -0.01175 & 0.11388 \\
\hline & 150 & 0.00993 & 0.06008 & -0.05017 & 0.06391 & 0.00211 & 0.06515 & 0.00013 & 0.10748 & 0.00591 & 0.06675 & -0.00696 & 0.10077 \\
\hline & 200 & 0.00640 & 0.05144 & -0.03953 & 0.05111 & 0.00054 & 0.05656 & 0.00456 & 0.10069 & 0.00327 & 0.05795 & -0.00103 & 0.09349 \\
\hline \multirow{4}{*}{2.0} & 30 & 0.04888 & 0.15100 & -0.06551 & 0.08322 & 0.01093 & 0.14490 & 0.00374 & 0.10534 & 0.02939 & 0.14953 & -0.01450 & 0.10745 \\
\hline & 50 & 0.02958 & 0.11242 & -0.04361 & 0.05646 & 0.00610 & 0.11333 & 0.00925 & 0.09198 & 0.01789 & 0.11492 & -0.00407 & 0.09061 \\
\hline & 150 & 0.01028 & 0.06031 & -0.01688 & 0.02267 & 0.00262 & 0.06316 & 0.01099 & 0.06825 & 0.00626 & 0.06325 & 0.00405 & 0.06398 \\
\hline & 200 & 0.00730 & 0.05147 & -0.01300 & 0.01767 & 0.00180 & 0.05421 & 0.01018 & 0.06153 & 0.00453 & 0.05420 & 0.00432 & 0.05713 \\
\hline \multirow{5}{*}{3.0} & 30 & 0.04836 & 0.15126 & -0.03689 & 0.04817 & 0.01270 & 0.14878 & 0.01132 & 0.07984 & 0.03287 & 0.15524 & -0.00436 & 0.08120 \\
\hline & 50 & 0.02892 & 0.11132 & -0.02377 & 0.03177 & 0.00798 & 0.11434 & 0.01107 & 0.06812 & 0.01981 & 0.11704 & 0.00015 & 0.06755 \\
\hline & 100 & 0.01375 & 0.07489 & -0.01269 & 0.01729 & 0.00424 & 0.07868 & 0.00793 & 0.05378 & 0.01002 & 0.07988 & 0.00153 & 0.05251 \\
\hline & 150 & 0.00848 & 0.06073 & -0.00869 & 0.01202 & 0.00164 & 0.06447 & 0.00699 & 0.04437 & 0.00555 & 0.06498 & 0.00264 & 0.04344 \\
\hline & 200 & 0.00624 & 0.05195 & -0.00674 & 0.00934 & 0.00170 & 0.05532 & 0.00439 & 0.03867 & 0.00464 & 0.05567 & 0.00116 & 0.03816 \\
\hline \multirow{5}{*}{5.0} & 30 & 0.05001 & 0.15442 & -0.01749 & 0.02376 & 0.01961 & 0.15907 & 0.00694 & 0.04931 & 0.04274 & 0.17055 & -0.00096 & 0.05659 \\
\hline & 50 & 0.02927 & 0.11350 & -0.01083 & 0.01488 & 0.01048 & 0.11861 & 0.00523 & 0.03872 & 0.02436 & 0.12622 & 0.00069 & 0.04530 \\
\hline & 100 & 0.01259 & 0.07560 & -0.00556 & 0.00768 & 0.00340 & 0.08071 & 0.00241 & 0.02570 & 0.01015 & 0.08429 & 0.00022 & 0.03082 \\
\hline & 150 & 0.01103 & 0.06227 & -0.00378 & 0.00529 & 0.00544 & 0.06732 & 0.00113 & 0.02034 & 0.01007 & 0.07002 & -0.00050 & 0.02405 \\
\hline & 200 & 0.00690 & 0.05248 & -0.00280 & 0.00395 & 0.00270 & 0.05750 & 0.00099 & 0.01760 & 0.00608 & 0.05969 & -0.00023 & 0.02060 \\
\hline
\end{tabular}

